\title{
The Effects of Dexamethasone in the Glycemic Control During Total Hip and Knee Arthroplasties: A Narrative Review
}

\author{
Brian H Goldman', Bradley Richey ${ }^{2}$, Brandon Foley², Alexandra Baker ${ }^{2}$, Justin J Toma ${ }^{3}$ and Pedro J Tort- Saade ${ }^{4}$ \\ ${ }^{1}$ Department of Orthopedic Surgery, Larkin Community Hospital, South Miami, FL, USA \\ ${ }^{2}$ University of Central Florida College of Medicine, Orlando, FL, USA \\ ${ }^{3}$ American University of Antigua College of Medicine, St. Johns, Antigua and Barbuda \\ ${ }^{4}$ Tort Orthopedic Institute, Professional Hospital, Guaynabo, Puerto Rico \\ *Corresponding Author: Brian H Goldman, Department of Orthopedic Surgery, Larkin Community Hospital, South Miami, FL, USA.
}

Received: September 20, 2019; Published: October 11, 2019

DOI: 10.31080/ASOR.2019.02.0111

\begin{abstract}
With increasing projected annual number of total joint replacement surgeries, postoperative standards of care are continuously being perfected. Dexamethasone is commonly used to enhance postoperative pain control, nausea, vomiting, and decrease the overall hospital length of stay. There is controversy regarding its usage in diabetic patients with concern for periprosthetic joint infection, postoperative hypoglycemia and ability to effectively manage postoperative pain and nausea. The current literature does not provide scientific evidence to determine whether dexamethasone affects glycemic control in diabetic patients. Moreover, it does not provide data on whether it is safe to use in diabetic patients during peri and postoperative management following total joint replacement. Keywords: Dexamethasone; Glycemic; Hip; Knee
\end{abstract}

\section{Abbreviations}

TJR: Total Joint Replacement; PONV: Post-Operative Nausea and Vomiting; TKA: Total Knee Arthroplasty; THA: Total Hip Arthroplasty; PJI: Periprosthetic Joint Infection; CRP: C-Reactive Protein; PMN: Polymorphonuclear; DM: Diabetes Mellitus; Hgb: Hemoglobin

\section{Introduction}

Total joint replacement (TJR) is one of the most effective treatments for late-stage joint degeneration from osteoarthritis, rheumatoid arthritis, osteonecrosis, and some complex traumatic joint injury. The last several decades have seen substantial increases in the frequency of TJR, with a $2.85 \%$ of people expected to receive TJR by 2050 [1]. In 2010 alone, over 243,000 TJR's were performed, carrying a substantial economic impact $[2,3]$. This commonly employed procedure involves resection and resurfacing of the damaged joint surface, followed by the implantation of a jointappropriate prosthetic device [4]. Patients receiving TJR are then followed for a period of several years by a multidisciplinary team to assist with rehabilitation and assess for comorbidities necessitating further surgical intervention.
Dexamethasone is a long-lasting glucocorticoid steroid and its use in TJR have been well characterized. Dexamethasone significantly reduces postoperative nausea and vomiting, as well as decreasing pain, hospital length of stay, and the necessity of opioid medications [5-7]. These effects have thus made it a mainstay of perioperative medical management for TJR. However, the use of strong glucocorticoids raises concerns for dangerous side effects. Some concerning side effects include reduced wound healing, hyperglycemia, and increased susceptibility to infection [8,9]. Hyperglycemia caused by dexamethasone is a controversial topic. There are studies that have demonstrated hyperglycemia caused by dexamethasone in both animal (rat) and human models [10,11]. However, other studies demonstrated that there was no significant difference in postoperative glucose levels nor change in glucose levels relative to baseline when comparing patients who did versus those who did not receive a single dose of dexamethasone perioperatively [12]. The concern regarding dexamethasone induced hyperglycemia stems from the fact that both diabetes and hyperglycemia are demonstrable risk factors for prosthetic joint infection (PJI) [13]. Due to the perceived risk of PJI, perioperative and postoperative dexamethasone use has yet to become standard of care in TJA [14]. Dexamethasone has been shown to mediate significant immuno- 
suppressive effects attributable to the upregulation of the CTLA- 4 ligand in naïve and mature $\mathrm{T}$ cells, attenuating CD-28 co-stimulation [15]. Despite this, in patients undergoing TJR, dexamethasone has been shown to not significantly increase the risk of PJI [7]. However, concerns of infection risk in certain patient subsets still exist. This review aims to analyze current literature regarding 1) perioperative utility of dexamethasone with regards to total knee arthroplasty (TKA) and total hip arthroplasty (THA), 2) risk of PJI associated with perioperative dexamethasone treatment, and 3) glucose level disruption in diabetic patients receiving perioperative dexamethasone injections.

\section{Dexamethasone uses in TJR}

In a study performed by Salerno., et al. $77 \%$ of patients undergoing a TJR experienced moderate-to-severe pain immediately postoperatively [16]. Poor pain control postoperatively has been directly correlated with decreased patient satisfaction, inferior outcomes, and longer hospital stays [17]. Thus, the use of paincontrol regimes including opioids, nerve blocks, and epidural anesthesia has become common in TJR [18]. However, the use of powerful opioids and pain management regimes has been shown to result in postoperative nausea and vomiting (PONV) [19]. Commonly, postoperative recovery following TJR begins with physical therapy on the first day after surgery but, considerable PONV may interfere with physical therapy activities. Incidences of PONV have been reported in at least $80 \%$ of patients undergoing TJR procedures with surgical anesthesia and no antiemetic prophylaxis, in one study [20].

The use of anti-emetics perioperatively has become common practice in TJR procedures. Several studies investigated dexamethasone's use and safety in orthopedic procedures and determined that it effectively reduced both pain and PONV in patients undergoing TJR procedures [21]. Kardash., et al. 's randomized control study of 50 patients undergoing THA found that in the patients receiving a single dose of dexamethasone perioperatively reported a significant reduction in pain with standing in just 24 hours postoperatively when compared to the control group receiving opioid-NSAID analgesia [22]. Additionally, the control group also demonstrated lower C-reactive protein (CRP) levels at 48 hours, which supported their hypothesis that single dose dexamethasone can have significant, measurable anti-inflammatory effects for at least 48 hours postoperatively [22].

Similarly, a randomized control study of 269 patients undergoing TKA, by Koh., et al. found that the preemptive administration of $10 \mathrm{mg}$ dexamethasone reduced postoperative pain as well as opioid consumption during the 6- to 24-hour period postoperatively and reduced overall opioid consumption during the entire 72-hour period [23].The control group received only ramose Tron, but the experimental group showed less overall opioid consumption and significantly lower pain visual analogue scale pain scores during the 6-24 hour postoperative period compared to the control. Additionally, Koh., et al. s study concluded that "prophylactic use of concurrent $10 \mathrm{mg}$ dexamethasone and ramose Tron reduced the overall incidence of PONV, rescue antiemetic requirement, improved the overall complete antiemetic response during the entire 72-hour evaluation period, and reduced the severity of nausea during the first 6-hours" [23].

\section{Infection}

Complications related to TJR occur in a minority of cases, with about $6 \%$ of cases requiring revision within 5 years [24]. However, these revision surgeries can be very costly. For revision TKA alone, the total economic burden currently is $\$ 2.7$ billion, and is expected to rise to over $\$ 13$ billion annually by 2030 [25]. Today, the most common etiology necessitating revision TJR surgery is postoperative infection, encompassing $20.4 \%$ of cases [25]. The Musculoskeletal Infection Society proposed the following definition for PJI following TJR. At least 1 major criteria, or 4 minor criteria must be met. Major criteria are sinus tract communication with the prosthesis, or 2 separate tissue positive tissue or fluid cultures from the affected joint. Minor criteria are 1) elevated erythrocyte sedimentation rate or CRP on labs, 2) elevated synovial white blood cell count, 3) elevated synovial polymorphonuclear (PMN) cells, 4) purulence of affected joint, 5) a positive tissue or fluid culture, and 6) $>5$ PMN cells per high powered field at 400x magnification [26]. It is therefore of vital importance to identify predisposing risk factors prior to the development of TJI.

Surgical revisions are the typical management of choice for PJIs, including debridement and/or one- or two-stage revision surgery, which are associated with further risk of re-infection [27]. Thus, it is crucial to avoid PJI in the initial TKA/THA due to the perceived effects it can have on future arthroplasties. A matched cohort study found that the risk of PJI increased three-fold in patients with a history of TKA or THA PJI with a ten-year cumulative incidence of $6.1 \%$ [28]. Another study found that patients with a history of diabetes, rheumatoid arthritis, steroid use, and prior joint surgery were also associated with increased risk of PJI [29]. With an estimated 4 million joint arthroplasties performed in the US by 2030, the prevalence of diabetes within this patient population (estimated to be at least $8 \%$ ) raises considerable concern in regards to PJI [30]. Marchant., et al. investigated the impact of glycemic control and diabetes mellitus (DM) on perioperative outcomes after TJA, stating there was a significantly increased risk of surgical site infection (adjusted odds ratio $=2.28 ; 95 \%$ confidence interval $=1.36$ to $3.81 ; \mathrm{p}=0.002$ ) and mortality in patients with uncontrolled DM (regardless of DM type) [31]. While this study found similar risk of PJI between patients with and without DM, none of the studies re- 
viewed in Marchant's analysis attempted to measure perioperative blood glucose levels, or their data was imprecise [32]. Although Marchant., et al. reported there was no correlation between DM and deep infections, patients with poor glycemic control had an infection rate 2.3 times greater than their counterparts [31]. Thus, it is reasonable to conclude that perioperative blood glucose level "is a more convincing predictor than the diagnosis of DM for PJI" [32].

It is important to note that inconsistencies are present in the current body of research. Merchant., et al. s result regarding uncontrolled DM and increased risk of PJI could not be replicated in recent articles that looked at glycemic control by hemoglobin (Hgb) A1c [33,34]. Additionally, the reliability of HgbA1c as a predictive measure of PJI after TJA remains controversial with Lorio., et al. reporting that $\mathrm{HgbA1c}$ levels are not reliable [34]. While the connection between hyperglycemia and PJI is also controversial, Yang., et al. 's meta-analysis of 6 retrospective studies including almost 27,000 patients concludes that high HgbA1c and perioperative hyperglycemia are associated with a significantly higher risk of PJI [32]. Thus, this association cannot be ignored and must be researched further bring light to the issue at hand.

\section{Glucose level disruption}

Glucose level is disrupted and increased with the use of dexamethasone. This effect is caused by the stimulation of gluconeogenesis in the liver resulting in an overall increase in blood glucose levels along with inhibition of glucose uptake by peripheral tissues. A study by Pasternak., et al. measured the effect of singledose dexamethasone on blood glucose during craniotomy. Dexamethasone was associated with significantly greater blood glucose concentration during each sampling interval than patients who received placebo [35]. Tien., et al. 2016 found that treatment of PONV with dexamethasone resulted in higher glucose levels than those receiving ondansetron in both DM and non-DM patients [36]. A study by Hans., et al. analyzed the effect of a single dexamethasone $10 \mathrm{mg}$ bolus on the blood glucose levels of both DM and nonDM patients undergoing abdominal surgery. Blood glucose concentrations remained significantly higher in the DM group. This study also concluded that the higher the patient's HgbA1c level, the greater the maximum blood glucose concentration was found to be. This also coincided with body mass index of the patient [37]. O'Connell., et al. did a retrospective review of $238 \mathrm{DM}$ patients who underwent TKA or THA. Patients who received dexamethasone had 4.07 (95\% CI: 2.46, 6.72) and 3.08 (95\% CI: 2.34, 4.04) higher odds of postoperative hyperglycemia in the first 24 and 72 hours respectively [38].

With only a handful of studies determining the effects of blood glucose following perioperative dexamethasone administration, further research is warranted on its effects especially when considering a DM patient. With many studies showing increased blood glucose levels, patients with diabetes should be looked more closely on whether to receive dexamethasone perioperatively. The risks of complications versus the benefits should be further warranted and more studies should be conducted with regards to complications occurring due to hyperglycemia.

\section{Discussion}

Total joint replacements are increasingly standard procedures. Thus, it is essential to optimize standards of postoperative care. The long-lasting glucocorticoid, dexamethasone, has proven useful in reducing postoperative nausea, vomiting, pain, length of hospital stays, and opioid use. However, it cannot be ignored that steroid use increases glucose levels which is a serious risk factor for PJI, one of the major complications of TJR. In some of the literature, postoperative dexamethasone has not shown increased risk of PJI (7) but in patients who already have hyperglycemia associated with DM this risk profile may not be the same [7]. According to the CDC, over 100 million United States adults are living with diabetes or prediabetes and $9.4 \%$ of the population has diagnostically confirmed diabetes [39]. Many patients undergoing TJR secondary to osteoarthritis are likely overweight which is one the strongest predictors of type 2 diabetes. Thus, diabetic patients are a major contributor to the TJR patient population, making dexamethasone's effects on blood glucose clinically important. Dexamethasone use in DM patients was associated with significantly greater blood glucose concentrations that remained greater for a longer period of time than non-DM patients [35,37]. Even independent of PJI, this risk of hyperglycemia does have implications for DM patients that may be clinically significant depending on disease severity.

Literature consensus on PJI and DM associations are harder to elucidate. In part, researchers have had a difficult time in deciding whether to monitor HbA1c, peri- and postoperative blood glucose, or simply preoperative DM diagnosis. Overall, it does seem that poor glycemic control leads to increased risk of surgical site infections and PJIs [29-34]. Often these result in revision surgeries which have a substantial economic burden, decrease patient quality of life, and expose patients to the risks of subsequent revisions.

With these risks, it is crucial to determine just how helpful and safe postoperative dexamethasone is. It has been proven to have measurable anti-inflammatory effects and reduce pain, opioid use, nausea, and vomiting [1,21-23]. Reducing these factors seems to allow for better adherence to early physical therapy and shorter hospital stays which likely increases patient satisfaction. However, it remains that there are other antiemetics and pain management protocols that could be explored as well. 


\section{Conclusion}

Dexamethasone has established itself as a useful medication following TJR concerning pain control, decreased length of hospital stay and PONV. However, many studies have established the association of dexamethasone with glucose level disruption, hyperglycemia linked PJI, and the higher risk of PJI in DM patients. The safety of dexamethasone in DM during TJR is unclear. Further studies focusing on the pain control, PONV, glucose level disruption in DM patients, and rate of PHI will better clarify the concerns surrounding dexamethasone administration during TJR procedures.

\section{Bibliography}

1. Inacio MCS., et al. "Projected increase in total knee arthroplasty in the United States - an alternative projection model". Osteoarthritis and Cartilage 25.11 (2017): 1797-1803.

2. Cram P., et al. "Total knee arthroplasty volume, utilization, and outcomes among Medicare beneficiaries, 1991-2010". Journal of the American Medical Association 308.12 (2012):12271236.

3. Losina E., et al. "Cost-effectiveness of total knee arthroplasty in the United States: patient risk and hospital volume". Archives of Internal Medicine 169.12 (2009):1113-1122.

4. Varacallo M JN. "Total Knee Arthroplasty (TKA) Techniques”. Statpearls Publishing (2019).

5. Vuorinen MA., et al. "Infection safety of dexamethasone in total hip and total knee arthroplasty: a study of eighteen thousand, eight hundred and seventytwo operations". International Orthopaedics 43.8 (2018): 1787-1792.

6. Klement MR., et al. "Intraoperative Dexamethasone Reduces Readmission Rates Without Affecting Risk of Thromboembolic Events or Infection After Total Joint Arthroplasty". The Journal of Arthroplasty 33.10 (2018): 3252-3256.

7. Richardson AB., et al. "Perioperative Dexamethasone Administration Does Not Increase the Incidence of Postoperative Infection in Total Hip and Knee Arthroplasty: A Retrospective Analysis". The Journal of Arthroplasty 31.8 (2016):1784-1787.

8. Wicke C., et al. "Effects of steroids and retinoids on wound healing". The Archives of Surgery 135.11 (2000):1265-1270.

9. Galandiuk S., et al. "The two-edged sword of large-dose steroids for spinal cord trauma”. Annals of surgery 218.4 (1993): 419-427.
10. Badmus 00 and Olatunji LA. "Glucocorticoid exposure causes disrupted glucoregulation, cardiac inflammation and elevated dipeptidyl peptidase-4 activity independent of glycogen synthase kinase-3 in female rats". Archives of Physiology and Biochemistry (2018): 1-9.

11. Alabbood M., et al. "Effect of high-dose dexamethasone on patients without diabetes during elective neurosurgery: a prospective study". Diabetology International 10.2 (2019): 109116.

12. Nurok M., et al. "Dexamethasone and perioperative blood glucose in patients undergoing total joint arthroplasty: A retrospective study". Journal of Clinical Anesthesia 37 (2017): 116122.

13. Mraovic B., et al. "Perioperative Hyperglycemia and Postoperative Infection after Lower Limb Arthroplasty". Journal of Diabetes Science and Technology 5.2 (2011): 412-418.

14. Godshaw BM., et al. "The Effects of Peri-Operative Dexamethasone on Patients Undergoing Total Hip or Knee Arthroplasty: Is It Safe for Diabetics?". The Journal of Arthroplasty 34.4 (2019): 645-649.

15. Giles AJ., et al. "Dexamethasone-induced immunosuppression: mechanisms and implications for immunotherapy". Journal for ImmunoTherapy of Cancer 6.1 (2018): 51.

16. Salerno A and Hermann R. "Efficacy and safety of steroid use for postoperative pain relief. Update and review of the medical literature". Journal of Bone and Joint Surgery 88.6 (2006): 1361-1372.

17. Capdevila X., et al. "Effects of perioperative analgesic technique on the surgical outcome and duration of rehabilitation after major knee surgery". Anesthesiology 91.1 (1999):8-15.

18. Singelyn FJ., et al. "Effects of intravenous patient-controlled analgesia with morphine, continuous epidural analgesia, and continuous three-in-one block on postoperative pain and knee rehabilitation after unilateral total knee arthroplasty". Anesthesia and Analgesia 87.1 (1998): 88-92.

19. Chen JJ., et al. "Efficacy of ondansetron and prochlorperazine for the prevention of postoperative nausea and vomiting after total hip replacement or total knee replacement procedures: a randomized, double-blind, comparative trial". Archives of Internal Medicine 158.19 (1998): 2124-2128. 
20. Kauste A., et al. "Droperidol, alizapride and metoclopramide in the prevention and treatment of post-operative emetic sequelae". European Journal of Anaesthesiology 3.1 (1986): 1-9.

21. Backes JR., et al. "Dexamethasone reduces length of hospitalization and improves postoperative pain and nausea after total joint arthroplasty: a prospective, randomized controlled trial". The Journal of Arthroplasty 28.8 (2013):11-17.

22. Kardash KJ., et al. "Single-dose dexamethasone reduces dynamic pain after total hip arthroplasty". Anesthesia and Analgesia 106.4 (2008):1253-1257.

23. Koh IJ., et al. "Preemptive low-dose dexamethasone reduces postoperative emesis and pain after TKA: a randomized controlled study". Clinical Orthopaedics and Related Research 471.9 (2013): 3010-3020.

24. Labek G., et al. "Revision rates after total joint replacement: cumulative results from worldwide joint register datasets". The Journal of Bone and Joint Surgery British 93.3 (2011):293297.

25. Delanois RE., et al. "Current Epidemiology of Revision Total Knee Arthroplasty in the United States". The Journal of Arthroplasty 32.9 (2017): 2663-2668.

26. Parvizi J., et al. "The 2018 Definition of Periprosthetic Hip and Knee Infection: An Evidence-Based and Validated Criteria". The Journal of Arthroplasty 33.5 (2018):1309-1314.e1302.

27. Kunutsor SK., et al. "Re-Infection Outcomes following Oneand Two-Stage Surgical Revision of Infected Hip Prosthesis: A Systematic Review and Meta-Analysis". PLoS One 10.9 (2015): e0139166.

28. Chalmers BP., et al. "Prior hip or knee prosthetic joint infection in another joint increases risk three-fold of prosthetic joint infection after primary total knee arthroplasty". The Bone and Joint Journal 101-B.7C (2019): 91-97.

29. Kunutsor SK., et al. "Patient-Related Risk Factors for Periprosthetic Joint Infection after Total Joint Arthroplasty: A Systematic Review and Meta-Analysis". PLoS One 11.3 (2016): e0150866.

30. Kurtz S., et al. "Projections of primary and revision hip and knee arthroplasty in the United States from 2005 to 2030". Journal of Bone and Joint Surgery 89.4 (2007):780-785.

31. Marchant M Jr., et al. "The impact of glycemic control and diabetes mellitus on perioperative outcomes after total joint arthroplasty". Journal of Bone and Joint Surgery 91.7 (2009):1621-1629.
32. Yang L., et al. "Is hemoglobin A1c and perioperative hyperglycemia predictive of periprosthetic joint infection following total joint arthroplasty? A systematic review and meta-analysis". Medicine 96.51 (2017): e8805-e8805.

33. Adams AL., et al. "Surgical outcomes of total knee replacement according to diabetes status and glycemic control, 2001 to 2009". Journal of Bone and Joint Surgery 95.6 (2013): 481-487.

34. Iorio R., et al. "Diabetes mellitus, hemoglobin A1C, and the incidence of total joint arthroplasty infection". The Journal of Arthroplasty 27.5 (2012):726-729.e721.

35. Pasternak JJ., et al. "Effect of single-dose dexamethasone on blood glucose concentration in patients undergoing craniotomy". Journal of Neurosurgical Anesthesiology 16.2 (2004):122125.

36. Tien M., et al. "The effect of anti-emetic doses of dexamethasone on postoperative blood glucose levels in non-diabetic and diabetic patients: a prospective randomised controlled study". Anesthesia 71.9 (2016):1037-1043.

37. Hans P., et al. "Blood glucose concentration profile after $10 \mathrm{mg}$ dexamethasone in non-diabetic and type 2 diabetic patients undergoing abdominal surgery". British Journal of Anaesthesia 97.2 (2006):164-170.

38. O'Connell RS., et al. "Dexamethasone and postoperative hyperglycemia in diabetics undergoing elective hip or knee arthroplasty: a case control study in 238 patients". Patient Safety in Surgery 12 (2018): 30.

39. Prevention CfDCa. "New CDC report: More than 100 million Americans have diabetes or prediabetes". CDC Newsroom Releases (2017).

\section{Volume 2 Issue 11 November 2019 (C) All rights arereserved by Brian H Goldman., et al.}

